\title{
Aprendizaje cooperativo y su influencia en las competencias digitales
}

Cooperative learning and its influence on digital skills

1 Héctor Daniel Morocho Lara

Universidad Técnica de Ambato, Facultad de Ciencias Humanas y de la Educación,

Carrera de Educación Básica. Ambato, Ecuador,

hd.morocho@uta.edu.ec

Artículo de Investigación Científica y Tecnológica

Enviado: 24/12/2021

Revisado: 29/12/2021

Aceptado: $12 / 01 / 2022$

Publicado:08/03/2023

DOI: $\underline{\text { https://doi.org/10.33262/concienciadigital.v6i1.4.2020 }}$

Morocho Lara, H. D. (2023). Aprendizaje cooperativo y su influencia en las competencias digitales. ConcienciaDigital, 6(1.4), 653-669. https://doi.org/10.33262/concienciadigital.v6i1.4.2020

CONCIENCIA DIGITAL, es una Revista Multidisciplinar, Trimestral, que se publicará en soporte electrónico tiene como misión contribuir a la formación de profesionales competentes con visión humanística y crítica que sean capaces de exponer sus resultados investigativos y científicos en la misma medida que se promueva mediante su intervención cambios positivos en la sociedad. https://concienciadigital.org _

La revista es editada por la Editorial Ciencia Digital (Editorial de prestigio registrada en la Cámara Ecuatoriana de Libro con No de Afiliación 663) www.celibro.org.ec 


\section{Palabras} claves: metodología, aprendizaje cooperativo, tic, competencias digitales.

Keywords: methodology, cooperative learning, tic, digital skills
Resumen

Introducción. Las tecnologías de la información y comunicación son mediadores culturales hacia un nuevo enfoque de comunidad educativa. El influjo de las tecnologías de la información y comunicación en las actividades de la vida social y sobre todo en el quehacer educativo ha superado condicionamientos basados en el antiguo "profesor sabelotodo y teórico". Objetivo. Este artículo de investigación tiene como objetivo describir y analizar el aporte del aprendizaje cooperativo para el desarrollo de competencias digitales, mediante la utilización de herramientas digitales de la web a través de una serie de programas y talleres vinculados a la aplicación de la tecnología. Metodología. La metodología implementada es una investigación de tipo experimental, mediante un cuestionario estructurado de 40 preguntas en escala de Likert el cual fue validado mediante el estadístico alfa de Cronbach con un resultado de 0,871 en la cual se trabajó con una población comprendida en dos grupos intactos; grupo control y grupo experimental, cada uno conformado por 30 sujetos. El contraste de la hipótesis se realizó mediante a prueba estadística de U de Mann-Whitney donde se obtuvo un valor menor a 0,05 , Resultados. por consiguiente, se obtuvo resultados favorables donde se afirma que el trabajo colaborativo aporta en el desarrollo de competencias digitales permitiendo un mayor desenvolvimiento de los individuos en actividades educativas diarias, Conclusión. Concluyendo pues que se garantiza un aprendizaje optimo y flexible dentro de un entono social, con ayuda y aplicación de las herramientas web 3.0.

\section{Abstract}

Introduction. Information and communication technologies are cultural mediators towards a new approach to the educational community. The influence of information and communication technologies in the activities of social life and especially in educational work has overcome conditions based on the old "know-it-all and theoretical teacher." Objective. This research article aims to describe and analyze the contribution of cooperative learning to the development of digital skills, through the use of digital tools on the web through a series of programs and workshops related to the application of technology. Methodology . The methodology implemented is an experimental type of 
investigation, through a structured questionnaire of 40 questions on a Likert scale which was validated by means of Cronbach's alpha statistic with a result of 0.871 in which we worked with a population comprised of two intact groups; control group and experimental group, each one made up of 30 subjects. The contrast of the hypothesis was performed using the Mann-Whitney U statistical test where a value less than 0.05 Results. Consequently, favorable results were obtained where it is stated that collaborative work contributes to the development of digital competences, allowing a greater development of individuals in daily educational activities, Conclusion. Concluding therefore that optimal and flexible learning is guaranteed within a social environment, with the help and application of web 3.0 tools.

\section{Introducción}

Durante generaciones, los profesionales de la educación han buscado un método óptimo de enseñanza y se han realizado considerables investigaciones comparando diferentes métodos docentes. Como bien planteaban en su estudio McKeachie (1986), el método de enseñanza más eficaz depende del objetivo, el estudiantado, el contenido y el profesorado, aunque la mejor respuesta es que "los estudiantes enseñan a otros estudiantes". El aprendizaje cooperativo es una estrategia de organización social de trabajo de los grupos y una metodología de enseñanza basada en la promoción de la cooperación caracterizada por la ausencia de competición (Pujolas, 2008).

En los contextos cooperativos, frente a los individualistas y competitivos, las tareas de unos y otros están interrelacionadas, es decir son interdependientes. Una de las características básicas del aprendizaje cooperativo es la interdependencia positiva en interacción cara a cara. La colaboración es una filosofía de la interacción y un estilo de vida personal en el cual los individuos son responsables de sus acciones, incluyendo el aprender y respetar las capacidades y las contribuciones de sus iguales (Damon \& Phelps, 1989).

En el aprendizaje cooperativo la responsabilidad del aprendizaje no recae en el profesorado como experto, sino que su rol se redefine de manera que pasa a ser también un estudiante. El alumnado es quien diseña su propia estructura de interacciones y mantiene el control en todo momento sobre las diferentes decisiones que repercuten en su aprendizaje, mientras que en el aprendizaje cooperativo es el docente quien diseña y mantiene casi por completo el control y la estructura de las interacciones que se han de dar en el grupo y los resultados finales que han de obtenerse. El aprendizaje cooperativo, en cambio, requiere una división de tareas entre los componentes del grupo que 
posteriormente se integran para la consecución del objetivo. Por otra parte, en el aprendizaje cooperativo se comparte la responsabilidad dándole mayor énfasis al proceso que a la tarea, de tal forma que se construye el conocimiento a través de la colaboración grupal (Doise, 2003).

Una optimización de la comunicación y de planteamientos humanistas entre todos los participantes y en todas las decisiones nos conduciría a un aprendizaje dialogado que se puede definir como aquel que se deriva del uso de competencias sociales y comunicativas donde los significados dependen de las interacciones humanas. Ofrece una propuesta de carácter interdisciplinar, es decir, es comunicacional, pedagógica, psicológica, sociológica y epistemológica (Flecha, 2009).

Teorías que fundamentan el aprendizaje cooperativo

a) Teoría de la interdependencia social Johnson \& Johnson (2008): la forma como se estructura la interdependencia social dentro de un grupo determina la interacción entre sus miembros. Básicamente, se establecen tres tipos de dinámicas interactivas en relación a las metas del grupo:

- Individualista: Cada alumno y alumna busca su propio beneficio sin tener en cuenta a los demás, porque no influyen unas metas sobre otras.

- Competitiva: El alumnado consigue sus objetivos, si los otros no lo alcanzan.

- Cooperativa: Cada alumno y alumna consigue sus objetivos si los demás alcanzan también los suyos. Los objetivos son comunes.

b) Teorías del desarrollo cognitivo, Clermont (2004): las capacidades humanas se desarrollan en interacción social.

Para la escuela de Ginebra el núcleo de todo proceso enseñanza- aprendizaje es la interacción social, ya que el conocimiento no se construye individualmente, sino que se construye cuando interactúan dos o más personas. La confrontación de puntos de vista moderadamente diferentes acerca de una misma tarea provoca un conflicto sociocognitivo que moviliza las estructuras intelectuales existentes y obliga a reestructurarlas, dando lugar al progreso intelectual (Lobato, 2008).

El ser humano, es el resultado de la interacción constante del individuo con el contexto sociocultural en que vive. Las funciones humanas, aparecen primero en un plano social y después se interiorizan. La zona de desarrollo próximo es la distancia entre el nivel de desarrollo real del niño o niña, determinado por la capacidad que muestra de forma independiente, y el nivel de desarrollo potencial, o capacidad mostrada bajo la orientación de un adulto o en colaboración con iguales más capacitados. La interacción es una sucesión de apoyos recíprocos que apunta a una construcción conjunta. Más allá de confrontar planteamientos, lo que sucede en la discusión es que lo que no se le ocurre a uno se le ocurre a otro, y esto da lugar a una construcción del razonamiento individual pero 
realizada de forma conjunta.

\section{Formas de aprendizaje cooperativo}

Los hermanos Johnson (1999), considerados los principales propulsores de esta metodología, a través de sus numerosas investigaciones, establecen tres formas de aprender cooperativamente: a través de grupos informales, grupos formales y grupos base. Aprendizaje cooperativo informal, son temporales, y se forman, para una situación concreta o para trabajar durante un período de una clase. El propósito es captar la atención del alumnado sobre los contenidos que deben aprender, establecer un contexto motivacional positivo, ayudar a organizar con antelación el material que va a cubrirse en la sesión. Los grupos formales de aprendizaje cooperativo pueden durar desde una clase hasta varias semanas para completar una tarea o encargo específico. En un grupo formal los estudiantes trabajan juntos para conseguir objetivos compartidos (Pujolas, 2004). Cada estudiante tiene dos responsabilidades: maximizar su aprendizaje y el de sus compañeros y compañeras de grupo.

\section{Ventajas del aprendizaje cooperativo}

Se conocen sus efectos y ventajas tanto en el desarrollo social como cognitivo de los estudiantes.

\section{Responde a las necesidades de una sociedad diversa y multicultural}

Se ha demostrado que, a través del aprendizaje cooperativo, se consigue una integración real del alumnado con dificultades de aprendizaje, debido a que se produce una mayor interacción positiva entre los alumnos y alumnas que se traduce en una mayor participación de todos y todas en la vida del aula.

\section{Contribuye al desarrollo cognitivo}

Consigue aumentar la variedad y la riqueza de experiencias que la escuela proporciona, ayudando a desarrollar mayores habilidades intelectuales y a mejorar la capacidad de expresión y comprensión verbal.

\section{Autoestima}

El aprendizaje cooperativo fomenta la autoestima de los estudiantes y la confianza en sí mismos, ya que les permite que se relajen y trabajen en un entorno tranquilo en el que encuentran el tiempo suficiente para pensar, las oportunidades para ensayar y recibir retroalimentación y, sobre todo, mayores probabilidades de éxito, derivadas tanto del apoyo o ayuda de sus compañeros y compañeras.

\section{Fomenta la interacción}


La interacción entre docentes y alumnado puede ser, en ocasiones, muy limitada, porque la interacción actual de nuestras aulas es muy elevada.

\section{Fomenta la autonomía e independencia}

Dentro de una dinámica cooperativa se reduce considerablemente la dependencia de los alumnos y alumnas con respecto al docente, ya que los compañeros y compañeras pueden proporcionar el tipo de apoyo que antes corría a cargo sólo del docente.

\section{Promueve el desarrollo de destrezas complejas de pensamiento crítico}

Cuando los estudiantes trabajan en contextos cooperativos, se ponen en juego toda una serie de destrezas metacognitivas relacionadas con la propia interacción cooperativa: planificación y organización de la tarea, toma de decisiones, argumentación y defensa de posturas, negociación de puntos de vista, resolución de problemas.

\section{Mejora el rendimiento académico}

Algunos de los factores que determinan que el aprendizaje cooperativo provoque un mayor rendimiento académico son:

\section{Competencias digitales}

En 2005 la Comisión Europea presentó una serie de recomendaciones sobre el aprendizaje permanente, proponiendo ocho competencias claves entre las que incluyó la competencia digital, que definió como el uso seguro y crítico de las tecnologías de la sociedad de la información para el trabajo, el ocio y la comunicación.

Se sustenta en las competencias básicas en materia de TIC: el uso de computadoras para obtener, evaluar, almacenar, producir, presentar e intercambiar información, y comunicarse y participaren redes de colaboración a través de internet. De este modo, es considerada, junto con otras siete competencias básicas, como aprendizaje imprescindible que los estudiantes deben alcanzar al terminar la escolarización obligatoria. La integración de las TIC en las aulas ha pasado de ser solo recomendable a hacerse imprescindible. Los tipos de habilidades que los ciudadanos necesitan están cambiando rápidamente y los sistemas de educación deben adaptarse para dotar a los jóvenes de las competencias necesarias.

Son algunas de las estrategias desde las que ha de partir la innovación educativa para hacer frente a la sociedad del siglo XXI. Adell (2004) sistematiza la integración de las TIC en el aula para el desarrollo de las competencias digitales en 5 puntos principales:

- Acceso: Aprender a utilizar correctamente la tecnología.

- Adopción: apoyar una forma tradicional de enseñar y aprender. 
- Adaptación: Integración en formas tradicionales de clase.

- Apropiación: uso colaborativo, proyectos y situaciones significativas.

- Innovación: Descubre nuevos usos de la tecnología y combinan las diferentes modalidades.

\section{Competencias TIC para el Aprendizaje}

"La capacidad de resolver problemas de información, comunicación y conocimiento, así como dilemas legales, sociales y éticos en ambiente digital” (Marqués, 2012).

\section{Dimensión Competencias digitales de búsqueda de información}

La dimensión búsqueda de información describe las habilidades para consultar, verificar, distinguir y clasificar información en entornos digitales y transformar o adaptar la información en un nuevo producto, conocimiento o desarrollar ideas nuevas. El docente debe estar capacitado para guiar al alumnado en su proceso de aprendizaje en el uso correcto y la integración didáctica de los nuevos contenidos, para capacitar al alumno en su recorrido futuro dentro de la llamada "sociedad del conocimiento" (Rangel, 2015).

\section{Dimensión Competencias digitales de comunicación}

La comunicación juega un rol importante en la preparación de estudiantes para ser no sólo aprendices sino también miembros de una comunidad más amplia, con voz y con la capacidad de hacer una contribución. Las habilidades incluidas en esta dimensión deben entenderse como habilidades sociales, donde la capacidad para transmitir e intercambiar información e ideas con otros, así como también de interactuar y contribuir dentro de un grupo o comunidad es fundamental (Escudero, 2009).

\section{Dimensión Competencias digitales de cooperación}

La cooperación juega un rol importante en la preparación de estudiantes para ser no sólo aprendices sino también miembros de una comunidad más amplia, con voz y con la capacidad de hacer una contribución. Esta dimensión describe las habilidades que se necesitan para trabajar a distancia. En particular, se define como la habilidad de negociar acuerdos dentro del respeto mutuo por las ideas del otro y de desarrollar contenidos con pares a distancia, utilizando distintos medios digitales (Kaye, 2012).

\section{Dimensión Competencias digitales de manejo de recursos web}

Esta dimensión define las habilidades funcionales y conocimientos necesarios para nombrar, resolver problemas, operar y usar las TIC en cualquier tarea. Es importante considerar que, por la permanente creación de software, hardware y programas, esta dimensión es particularmente dinámica (Seale, 2009). 
Esta dimensión se centra en:

Conocimientos TIC: se refiere a la capacidad de manejar y entender conceptos TIC utilizados para nombrar las partes y funciones de los computadores y las redes. Dominar los términos asociados a las TIC y sus componentes es importante para poder resolver problemas técnicos asociados a ellas.

Operar las TIC: considera la capacidad de usar las TIC de forma segura, de resolver problemas técnicos básicos y de administrar información y archivos.

Usar las TIC: se refiere a la habilidad de dominar software, hardware y programas de uso extendido en la sociedad, particularmente aquellos que facilitan el aprendizaje individual y con otros.

Tabla 1

Matriz de competencias digitales

\begin{tabular}{|c|c|}
\hline DIMENSIONES & INDICADORES \\
\hline & Definir \\
\hline \multirow[t]{8}{*}{ COMPETENCIAS DE BÚSQUEDA DE INFORMACIÓN } & Generar \\
\hline & Elegir \\
\hline & Ordenar \\
\hline & Estructurar \\
\hline & Especificar \\
\hline & Combinar \\
\hline & Verificar \\
\hline & Diseñar \\
\hline \multirow[t]{5}{*}{ COMPETENCIAS DE COMUNICACIÓN } & Reconocer \\
\hline & Aplicar \\
\hline & Comunicar \\
\hline & Elaborar \\
\hline & Destacar \\
\hline \multirow[t]{5}{*}{ COMPETENCIAS DE COOPERACIÓN } & Intercambiar \\
\hline & Debatir \\
\hline & Acordar \\
\hline & Desarrollar \\
\hline & Publicarlos \\
\hline \multirow[t]{5}{*}{ COMPETENCIAS DE MANEJO DE RECURSOS WEB } & Entendimiento conceptual - práctico \\
\hline & Diagnostico \\
\hline & Aplicar \\
\hline & Resolver \\
\hline & Utilizar \\
\hline
\end{tabular}




\section{Metodología}

La investigación desarrollada enmarcada en el enfoque cuantitativo, estudio aplicado de tipo transversal, para Hernández et al. (2016), el diseño de esta investigación es cuasi experimental porque se manipuló intencionadamente la variable independiente para observar su consecuencia sobre la variable dependiente. Se trabajó con dos grupos intactos (ya estaban formados antes del experimento). Para la obtención de la muestra se aplicó el tipo de muestreo no probabilístico intencional, seleccionándose a criterio del investigador a 60 estudiantes, divididos en dos grupos, uno de experimentación y el otro de control. En esta investigación se utilizó el método Hipotético-Deductivo (InducciónDeducción) porque es un estudio experimental. Se partió de la observación para plantear un problema. Mediante un proceso de inducción este problema se remitió a una teoría. Partiendo del marco teórico se planteó una hipótesis, mediante un razonamiento deductivo, se validó empíricamente.

La técnica para el acopio de datos utilizada en el presente trabajo de investigación fue la encuesta, para calificar las competencias digitales, con los criterios establecidos

Los instrumentos que se aplicaron para recoger información fueron:

1.- Pre test de conocimientos: Este instrumento se aplicó al inicio de la investigación a todos los estudiantes que conforman el grupo de experimentación y de control, cuyo objetivo fue determinar el nivel de conocimientos que tenía los dicentes en relación a competencias digitales.

2.- Pos test de conocimientos. - Esta prueba se utilizó al final de la investigación, un postest fue dirigido al grupo de experimentación a quienes se les aplico el programa de estrategias de aprendizaje cooperativo para el desarrollo de competencias digitales y el mismo instrumento fue aplicado al grupo de control a quienes no se les aplico el programa de aprendizaje.

Para determinar la eficacia del programa de aprendizaje para la elaboración del proyecto de investigación, se realizó un análisis comparativo los resultados de las encuestas obtenidas por los estudiantes pertenecientes al grupo de experimentación y de control.

La validación del instrumento de investigación se realizó a través de la evaluación y criterios externos de expertos que analizaron el instrumento y determinaron si el instrumento es válido o no para medir la variable.

Dada la validez del instrumento por Juicio de Expertos, donde el cuestionario sobre competencias digitales obtuvo el valor de $100 \%$, se puede deducir que el cuestionario tiene un nivel de validez Excelente encontrarse dentro del rango respectivo. 
Con el objetivo de valorar la consistencia interna del instrumento se procedió a calcular el alfa de Cronbach para el total de la muestra conformada por estudiantes pertenecientes a la Carrera de Ciencias Exactas, paralelos "A" y "B" de la Facultad de Ciencias de la Educación, Humanas y Tecnologías de la Universidad Nacional de Chimborazo, resultando en 0,871 por lo que la fiabilidad de la escala se puede considerar muy alta, considerando el contenido del instrumento en las competencias de: Búsqueda de información, de comunicación, de cooperación y manejo de recursos web.

El análisis estadístico se hizo mediante procesos estadísticos descriptivos y procesos estadísticos inferenciales. Se utilizó el paquete computarizado SPSS 23.

Se aplicó análisis descriptivos, porcentaje en la variable y sus dimensiones, su representación mediante diagramas de barras.

En esta investigación se utilizó el estadístico U de Mann Whitney que es una prueba no paramétrica aplicada a dos muestras independientes.

\section{Resultados}

El cuestionario de competencias digitales conformado por 40 ítems, que comprende las siguientes dimensiones

Dimensión competencias búsqueda de la información: 10 ítems

Dimensión competencia de comunicación: 10 ítems

Dimensión competencia de cooperación:10

ítems Dimensión manejo de recursosweb:10

ítems

Baremos

- Competencias digitales: Inicio [40-80] Proceso [81-120] Logrado [121- 160]

- Dimensión competencias búsqueda de la información: Inicio [10-20] Proceso [21-30] Logrado [31-40]

- Dimensión competencia de comunicación: Inicio [10-20] Proceso [21-30] Logrado[31-40]

- Dimensión competencia de cooperación: Inicio [10-20] Proceso [21-30] Logrado [31-40]

- Dimensión manejo de recursos web: Inicio [10-20] Proces o [21-30] Logrado [31-40] 
Tabla 2

Descripción de los niveles de la competencia búsqueda de información

\begin{tabular}{lclllllll}
\hline & \multicolumn{2}{l}{ GC Pre test } & \multicolumn{2}{l}{ GC Post test } & \multicolumn{2}{c}{ GE Pre test } & \multicolumn{2}{c}{ GE Post test } \\
\hline & $\mathrm{f}$ & $\%$ & $\mathrm{f}$ & $\%$ & $\mathrm{f}$ & $\%$ & $\mathrm{f}$ & $\%$ \\
\hline Logrado & 0 & $0 \%$ & 0 & $0 \%$ & 0 & $0 \%$ & 27 & $90 \%$ \\
\hline Proceso & 3 & $10 \%$ & 23 & $76.7 \%$ & 1 & $3.3 \%$ & 3 & $10 \%$ \\
\hline Inicio & 27 & $90 \%$ & 7 & $23.3 \%$ & 29 & $96.7 \%$ & 0 & $0 \%$ \\
\hline Total & 30 & $100,0 \%$ & 30 & $100,0 \%$ & 30 & $100,0 \%$ & 30 & 100 \\
\hline
\end{tabular}

Fuente: Cuestionario aplicado a los estudiantes

\section{Figura 1}

\section{Porcentaje de la competencia búsqueda de la información}

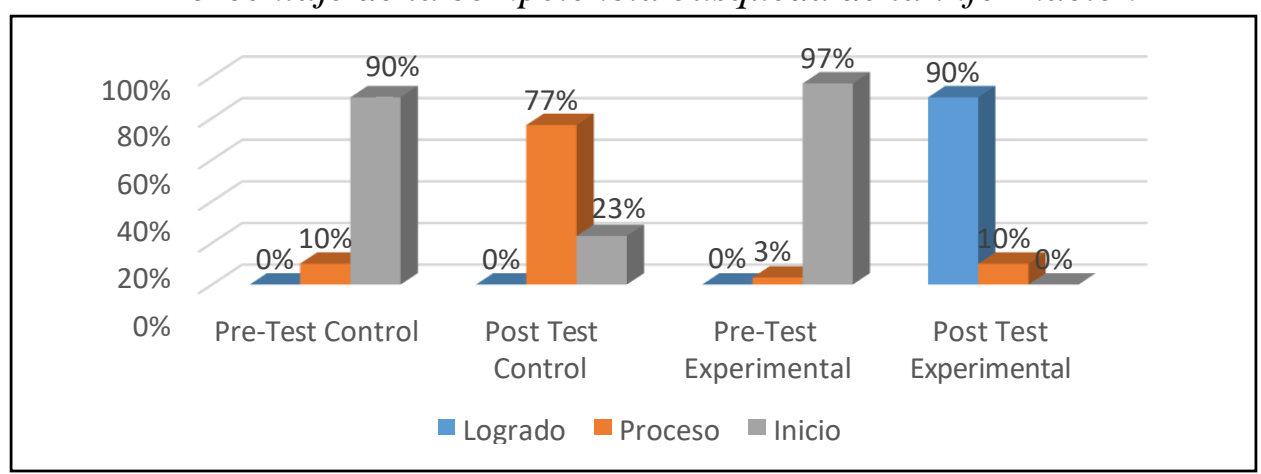

Fuente: Cuestionario aplicado a los estudiantes

Los datos de la tabla 2 y figura 1 respectiva muestran que en el pretest en el grupo control el $90 \%$ se encuentra en el nivel inicio y el $10 \%$ en el nivel proceso, en cuanto al grupo experimental $97 \%$ en el nivel inicio mientras que el $3 \%$ proceso.

En cuanto al pos test en el grupo control el $23 \%$ se encuentra en el nivel de inicio, el $77 \%$ en el nivel proceso mientras que en el grupo experimental el $10 \%$ se encuentra en el nivel proceso y el $90 \%$ en el nivel logrado en cuanto a la competencia búsqueda de la información.

Tabla 3

Descripción de los niveles de la competencia de comunicación

\begin{tabular}{lccllllll}
\hline & \multicolumn{2}{c}{ GC Pre test } & \multicolumn{2}{c}{ GC Post test } & \multicolumn{2}{c}{ GE Pre test } & \multicolumn{2}{c}{ GE Post test } \\
\hline & $\mathrm{f}$ & $\%$ & $\mathrm{f}$ & $\%$ & $\mathrm{f}$ & $\%$ & $\mathrm{f}$ & $\%$ \\
\hline Logrado & 0 & $0 \%$ & 0 & $0 \%$ & 0 & $0 \%$ & 23 & $76.7 \%$ \\
\hline Proceso & 1 & $3.3 \%$ & 22 & $73.3 \%$ & 3 & $10 \%$ & 7 & $23.3 \%$ \\
\hline Inicio & 29 & $96.7 \%$ & 8 & $26.7 \%$ & 27 & $90 \%$ & 0 & $0 \%$ \\
\hline
\end{tabular}




\begin{tabular}{|c|c|c|c|c|c|c|c|c|}
\hline Total & 30 & $100,0 \%$ & 30 & $100,0 \%$ & 30 & $100,0 \%$ & 30 & $100,0 \%$ \\
\hline
\end{tabular}

Figura 2

Porcentaje de la competencia de comunicación

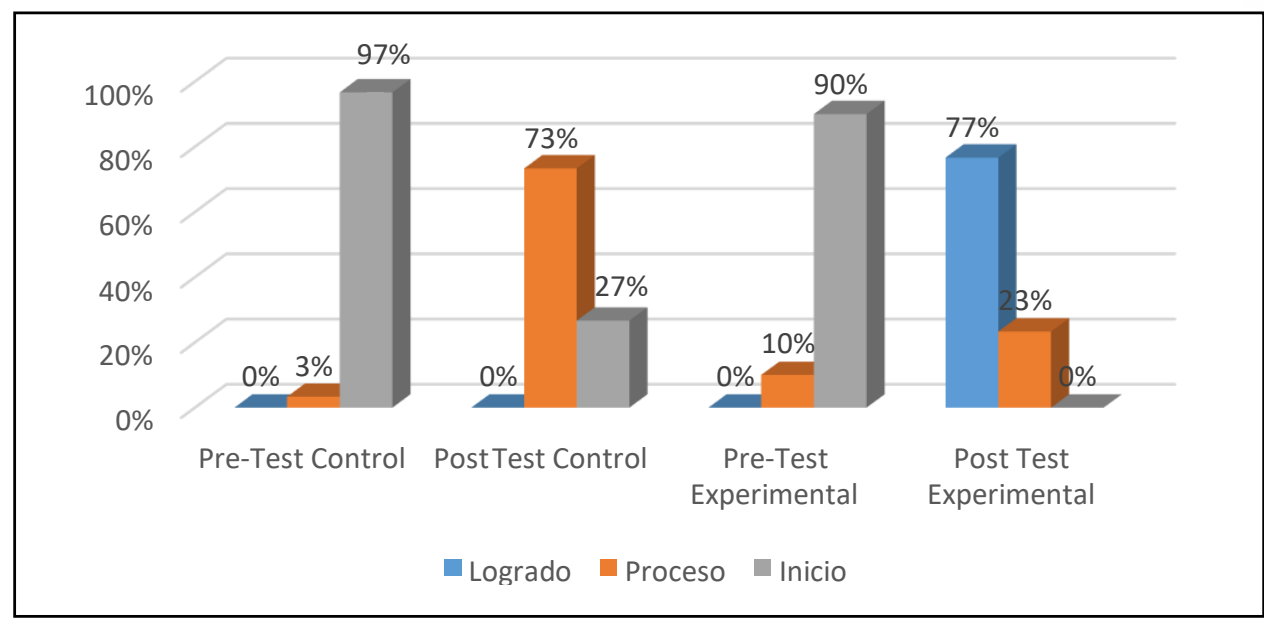

Fuente: Cuestionario aplicado a los estudiantes

Los datos de la tabla 3 y figura 2 respectiva muestran que en el pretest en el grupo control el $97 \%$ se encuentra en el nivel inicio y el $3 \%$ en el nivel proceso, en cuanto al grupo experimental $90 \%$ en el nivel inicio mientras que el $10 \%$ proceso.

En cuanto al pos test en el grupo control el $27 \%$ se encuentra en el nivel de inicio, el $73 \%$ en el nivel proceso mientras que en el grupo experimental el $23 \%$ se encuentra en el nivel proceso y el $77 \%$ en el nivel logrado en cuanto a la competencia de comunicación.

\section{Tabla 4}

Descripción de los niveles de la competencia de cooperación

\begin{tabular}{lcccccccc}
\hline & \multicolumn{2}{l}{ GC Pre test } & \multicolumn{2}{l}{ GC Post test } & \multicolumn{2}{l}{ GE Pre test } & \multicolumn{2}{c}{ GE Post test } \\
& $\mathrm{f}$ & $\%$ & $\mathrm{f}$ & $\%$ & $\mathrm{f}$ & $\%$ & $\mathrm{f}$ & $\%$ \\
\hline Logrado & 0 & $0 \%$ & 0 & $0 \%$ & 0 & $0 \%$ & 30 & $100 \%$ \\
\hline Proceso & 0 & $0 \%$ & 19 & $63.3 \%$ & 0 & $0 \%$ & 0 & $0 \%$ \\
\hline Inicio & 30 & $100 \%$ & 11 & $36.7 \%$ & 30 & $100 \%$ & 0 & $0 \%$ \\
\hline Total & 30 & $100,0 \%$ & 30 & $100,0 \%$ & 30 & $100,0 \%$ & 30 & 100,0 \\
\hline
\end{tabular}

Fuente: Cuestionario aplicado a los estudiantes 


\section{Figura 3}

Porcentaje de la competencia de cooperación

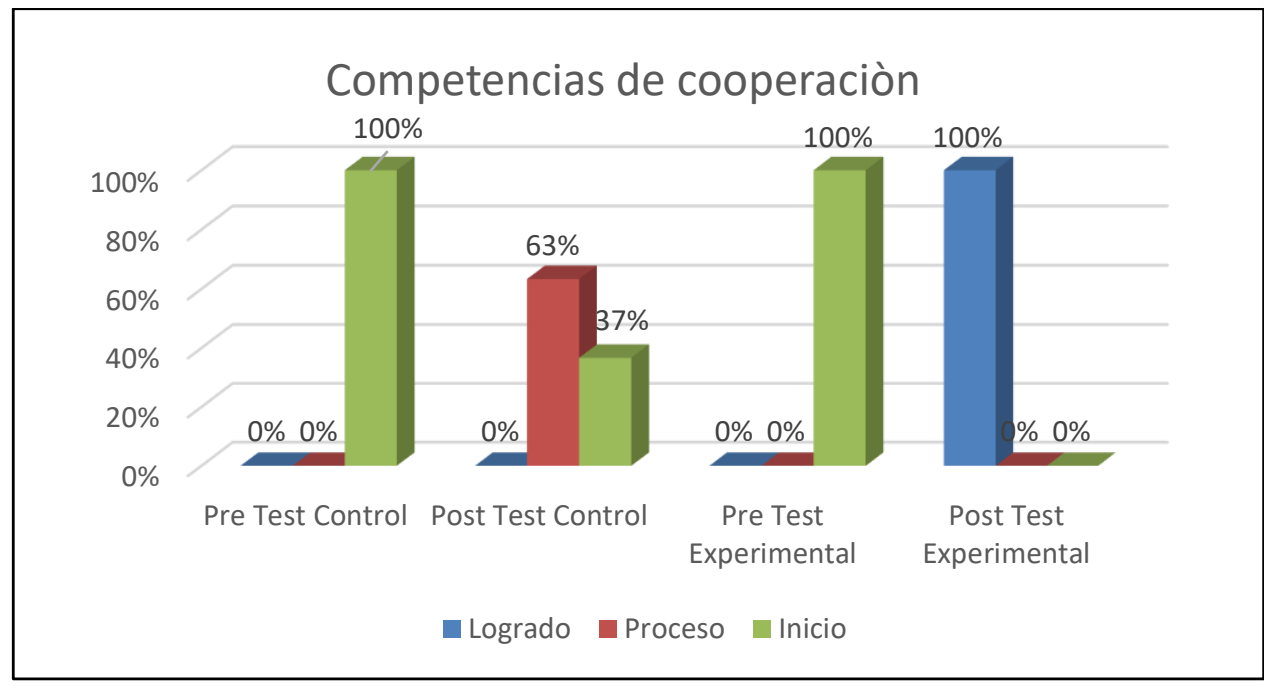

Fuente: Cuestionario aplicado a los estudiantes

Los datos de la tabla 4 y figura 3 respectiva muestran que en el pretest en el grupo control el 100\% se encuentra en el nivel inicio, en cuanto al grupo experimental $100 \%$ en el nivel inicio. En cuanto al pos test en el grupo control el 37\% se encuentra en el nivel de inicio, el $63 \%$ en el nivel proceso mientras que en el grupo experimental el 100\% en el nivel logrado en cuanto a la competencia de cooperación.

\section{Tabla 5}

Descripción de los niveles de la competencia de manejo de recursos web

\begin{tabular}{lcccccccc}
\hline & \multicolumn{2}{l}{ GC Pre test } & \multicolumn{2}{l}{ GC Post test } & \multicolumn{2}{c}{ GE Pre test } & \multicolumn{2}{c}{ GE Post test } \\
& $\mathrm{f}$ & $\%$ & $\mathrm{f}$ & $\%$ & $\mathrm{f}$ & $\%$ & $\mathrm{f}$ & $\%$ \\
\hline Logrado & 0 & $0 \%$ & 0 & $0 \%$ & 0 & $0 \%$ & 28 & $93.3 \%$ \\
\hline Proceso & 3 & $10 \%$ & 20 & $66.7 \%$ & 2 & $6.7 \%$ & 2 & $6.7 \%$ \\
\hline Inicio & 27 & $90 \%$ & 10 & $33.3 \%$ & 28 & $93.3 \%$ & 0 & $0 \%$ \\
\hline Total & 30 & $100,0 \%$ & 30 & $100,0 \%$ & 30 & $100,0 \%$ & 30 & $100,0 \%$ \\
\hline
\end{tabular}

Fuente: Cuestionario aplicado a los estudiantes 


\section{Figura 4}

Porcentaje de la competencia de cooperación

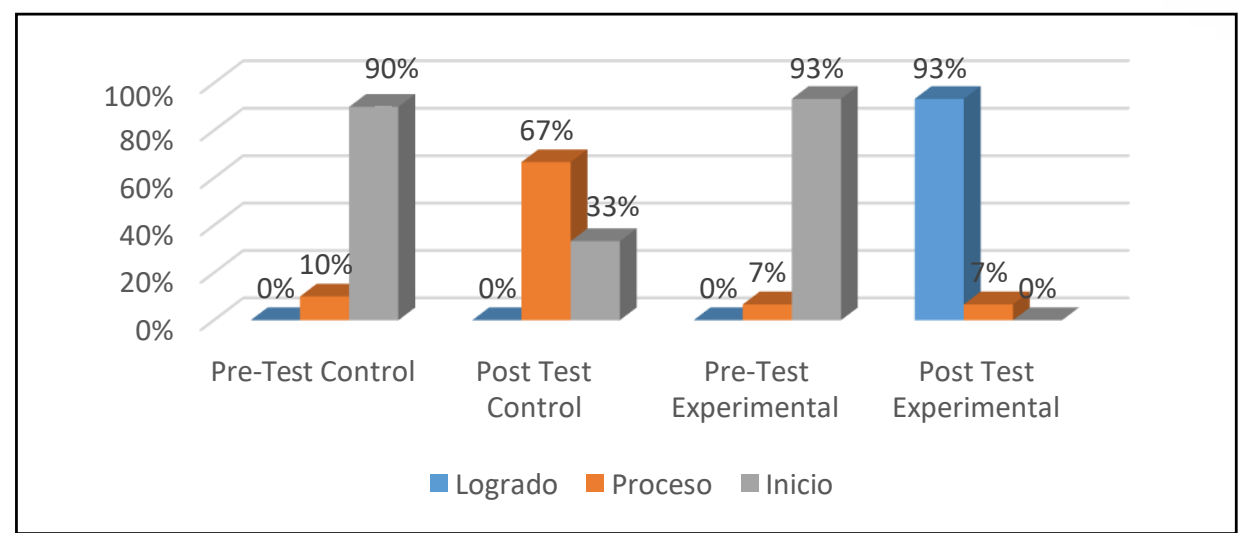

Fuente: Cuestionario aplicado a los estudiantes

Los datos de la tabla 5 y figura 4 respectiva muestran que en el pretest en el grupo control el $90 \%$ se encuentra en el nivel inicio y el $10 \%$ en el nivel proceso, en cuanto al grupo experimental $93 \%$ en el nivel inicio y el $7 \%$ en el nivel de proceso.

En cuanto al pos test en el grupo control el 33\% se encuentra en el nivel de inicio, el $67 \%$ en el nivel proceso mientras que en el grupo experimental el $93 \%$ en el nivel logrado y el $7 \%$ en el nivel proceso, en cuanto a la competencia de manejo de recursos web.

Los resultados que se muestran a continuación se basan en las preguntas más representativas seleccionadas dentro de la experimentación. De esta forma es cómo se realiza el cálculo del estadístico de Kolmogórov-Smirnov en donde se pudo comprobar la hipótesis.

\section{Tabla 6}

Test de Kolmogórov-Smirnov

\begin{tabular}{lllll}
\hline \hline Rangos & & & & \\
& Grupo & $\mathrm{N}$ & Rango promedio & Suma de rangos \\
\cline { 2 - 5 } Pre test & Control & 30 & 28,85 & 865,50 \\
& Experimental & 30 & 32,15 & 964,50 \\
& Total & 60 & & \\
Pos test & Control & 30 & 15,50 & 465,00 \\
& Experimental & 30 & 45,50 & 1365,00 \\
& Total & 60 & & \\
\hline \hline
\end{tabular}


Tabla 7

Test de Kolmogórov -Smirnov

\begin{tabular}{lll}
\hline Estadísticos de prueba $^{\mathrm{a}}$ & & \\
\hline \hline U de Mann-Whitney & Pre test & Pos test \\
W de Wilcoxon & 400,500 &, 000 \\
Z & 865,500 & 465,000 \\
Sig. asintótica (bilateral) &, 462 & $-6,657$ \\
\hline \hline
\end{tabular}

Como se observa en los datos no existe diferencias significativas entre el grupo control y experimental en el pretest, mientras que si existen diferencias significativas entre el grupo control y experimental en el pos test teniendo mayor ventaja los estudiantes del grupo experimental.

En la prueba U de Mann Whitney en el pos-test el valor de $\rho=0,000<0,05$ se rechaza la hipótesis nula y se acepta la hipótesis alterna que es: El aprendizaje cooperativo influye significativamente en las competencias digitales de los estudiantes de Ciencias Exactas de la Universidad Nacional de Chimborazo.

\section{Conclusiones}

- Se ha demostrado que existe una influencia significativa de la aplicación del aprendizaje cooperativo en competencias digitales de los estudiantes, según Mann Whitney de 0,000 y siendo altamente significativo, rechaza la hipótesis nula y tenemos que: La aplicación del aprendizaje cooperativo influye significativamente en competencias digitales de los estudiantes de Ciencias Exactas de la Universidad Nacional de Chimborazo.

- Se ha verificado que existe una influencia significativa de la aplicación del aprendizaje cooperativo en competencias de búsqueda de información de los estudiantes, según Mann Whitney de 0,000 y siendo altamente significativo tenemos que: La aplicación del aprendizaje cooperativo influye significativamente en competencias de búsqueda de información de los estudiantes de Ciencias Exactas de la Universidad Nacional de Chimborazo.

- Se ha evidenciado que existe una influencia significativa de la aplicación del aprendizaje cooperativo en competencias de comunicación de los estudiantes, según Mann Whitney de 0,000 y siendo altamente significativo tenemos que: La aplicación del aprendizaje cooperativo influye significativamente en 
competencias de comunicación de los estudiantes de Ciencias Exactas de la Universidad Nacional de Chimborazo.

\section{Referencias bibliográficas}

Adell, J. (2004). Internet en Educación. Comunicación y pedagogía. EDUTEC, 25-28.

Clermont, P. (2004). La construcción de la inteligencia en la interacción social. Valencia: Visor.

Damon, W. \& Phelps, E. (1989). Critical distinctions among three approaches to peer education. International Journal of Educational Research, 13(1), 9-19.

Doise, W. \& Mugny, G. (2003). La construcción social de la inteligencia. México: Trillas.

Escudero, J. (2009). La integración de las nuevas tecnologías en el curriculum y en el sistema escolar. Tecnología educativa, 397-412.

Flecha, R. (2009). Aprendizaje dialógico en la sociedad de la información. España: Paidós.

Hernández, R., Fernández C \& Baptista L. (2016). Metodología de la investigación. México: McGrawHill.

Johnson. (1999). Aprender juntos y solos. United States: AIQUE.

Johnson, J. \& Johnson. (2008). Cooperative learning y Social Interdependence Theory. United States: AIQUE.

Kaye, A. R. (2012). Collaborative learning through computer conferencing: The Najaden papers 90. Nueva York: Springer Science y Business media.

Lobato, C. (2008). El trabajo en grupo. Aprendizaje cooperativo en secundaria. Bilbao: Euska.

Marqués, P. (2012). Competencias básicas en la sociedad de la información. Bilbao: Euska.

McKeachie. (1986). Teaching y learning in the college classroom. United Stated: Cengage learning.

Pujolas, P. (2008). El aprendizaje cooperativo. Barcelona. Graó.

Pujolas, P. (2004). Aprender juntos alumnos diferentes. Los equipos de aprendizaje cooperativo. Barcelona. Eumo-octaedro. 
Rangel, A. (2015). Competencias docentes digitales: propuesta de un perfil. Medios y educación, 235-248.

Seale, J. (2009). Learning technology in transition. Lisse - Netherlands: Swets.

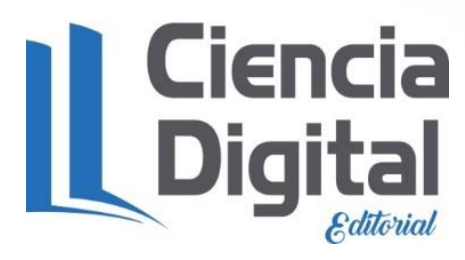

El artículo que se publica es de exclusiva responsabilidad de los autores y no necesariamente reflejan el pensamiento de la Revista Conciencia Digital.

\section{Ciencia
Digital
Eutuolal}

El artículo queda en propiedad de la revista y, por tanto, su publicación parcial y/o total en otro medio tiene que ser autorizado por el director de la Revista Conciencia Digital.
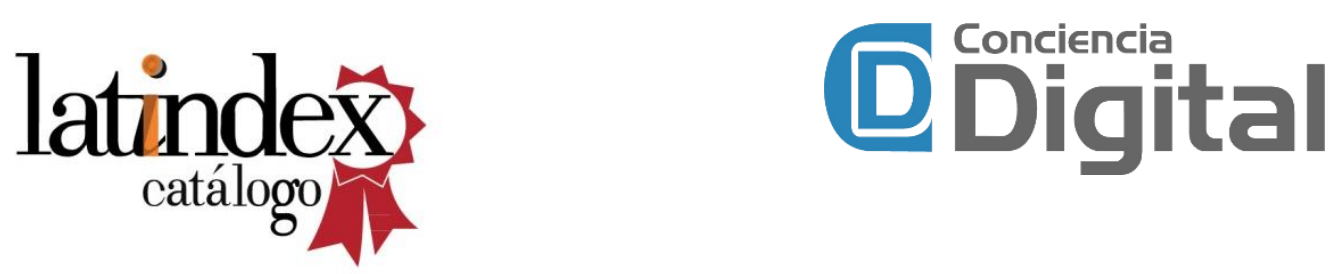

Indexaciones

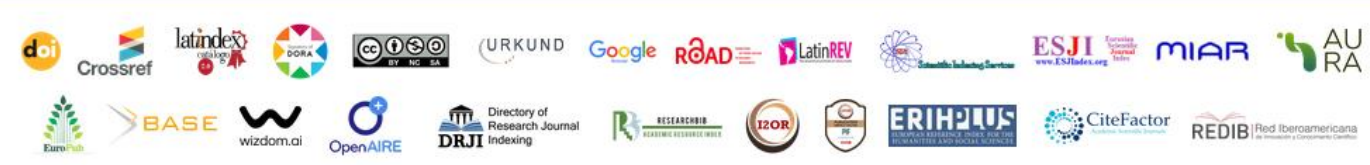

